und dünner ausgezogenen, als Stechheber dienenden Rohres eingefüllt, dann die Röhren $b$ abgeschmolzen.

Auffindung flüchtiger sâuren. Bekanntlich bietet das Zusammenschmelzen einer Substanz mit saurem schwefelsauren Kali häufig ein einfaches und bequemes. Mittel, flüchtige Säuren, Jod etc. aus Verbindungen auszutreiben und an der Farbe, dem Geruch oder der Wirkung auf Reagentien zu erkennen. Pis a ni (Annal. des Mines, $5^{\text {me }}$ Sér. 19. 477) empfiehlt zu diesem Zwecke einen kleinen, netten Apparat. Das Zusammenschmelzen geschieht in einem 6-8 Centimeter langen Proberöhrchen, welches mittelșt eines Pfropfes mit einem kleinen dem V a r r e ntrapp-Will'schen Absorptionsapparat ähnlichen Kugelrohre verbunden ist. In die Kugel des letzteren bringt man das der Wirkung der Dämpfe auszusetzende Reagens : einige Tropfen Kalkwasser für Kohlensäure, Eisenvitriollösung für salpetrige Dämpfe, Kalilauge für Cyanverbindungen etc. Zur Entdeckung der Chlorverbindungen dient das bekannte auf die Erzeugung der Chlorchromsäure hinzielende Verfahren, Brom und Jod werden an der Farbe ihrer Dämpfe erkannt, Oxalsäure an dem gleichzeitigen Auftreten von Kohlensäure und Kohlenoxyd. Das letztere wird, aus einer kleinen Spitze ausströmend, angezündet, nachdem man es zuerst durch ein kleines, etwas Kalihydrat enthaltendes Röhrchen geleitet hat.

Ich ziehe es häufig vor, das Zusammenschmelzen in einem kleinen Platin- oder Porzellanschiffchen vorzunehmen, welches in eine horizontale Glasröhre eingeschoben ist. . Durch das eine Ende derselben leitet man reine atmosphärische Luft in ganz langsamem Strome ein, das andere verbindet man mit einem kleinen U-förmigen Röhrchen, welches durch das eingegossene flüssige Reagens eben abgesperrt ist. Es lässt sich auf diese Weise namentlich auch Fluor in durch Schwefelsäure zersetzbaren kieselsäurehaltigen Mineralien ermitteln. Als Reagens dient wässriges Ammoniak; es bildet sich, unter Ausscheidung von Kieselsäurehydrat, Fluorammonium.

\title{
Beiträge zur näheren Kenntniss des Sauerstoffs und der einfachen
} Salzbildner. Unter dieser Ueberschrift hat Schönbein (Journ., f. prakt. Chem. 84. 385) eine Reihe von Versuchen mitgetheilt, wodurch die herrschenden Ansichten in Betreff sehr einfacher Reactionen mannigfach berichtigt und viele neue Thatsachen aufgedeckt werden. Ich theile im Nachstehenden, dem Plane der Zeitschrift gemäss, die Reactionen mit, welche mit der analytischen Chemie direct oder indirect in Beziehung stehen, verweise aber in Betreff der interessanten theoretischen 
Auseinandersetzungen, welche der Verfasser daran knüpft, auf die Originalabhandlung.

1. Chlorwasser, mit wässrigem Ammoniak bis zur alkalischen Reaction versetzt, liefert eine Flüssigkeit, welche alle Reactionen unterchlorigsaurer Alkalien zeigt. Erst allmählich verrliert die Flüssigkeit unter merklicher Entbindung von Stickgas diese Eigenschaften, langsamer in der Kälte, rascher bei erhöhter Temperatur. Es ist somit wahrscheinlich, dass zuerst nur Chlorammonium und unterchlorigsaures Ammoniumoxyd entstehen, welches letztere alsdann allmählich nach der Gleichung $3\left(\mathrm{NH}_{4} \mathrm{O}, \mathrm{ClO}\right)=2 \mathrm{~N}+\mathrm{NH}_{4} \mathrm{Cl}+2 \mathrm{HCl}+6 \mathrm{HO}$ zerfällt. - Neben dieser Einwirkung findet noch eine zweite Statt, welche etwas chlorsaures Ammon entstehen lässt.

2. Bromwasser und Jodwasser zeigen ganz dasselbe Verhalten wie Chlorwasser. Bei Anwendung eines möglichst concentrirten Jodwassers bildet sich auch eine kleine Menge des sogenannten Jodstickstoffs.

3. Tröpfelt man zu einer gesättigten Lösung von Jod in Wasser Kalilauge, bis die Reaction dentlich alkalisch geworden, so dass sie weiteres Jodwasser augenblicklich entfärben würde, so bläut die Flüssigkeit reinen Kleister, noch tiefer jodkaliumhaltigen, bräunt Jodkaliumlösung, entfärbt Indigotinctur und zeigt einen eigenthümlichen safranähnlichen Geruch. Bei niedriger Temperatur büsst sie diese Eigenschaften allmählich, beim Sieden rasch ein. Die gleichen Eigenschaften, nur in erhöhtem Grade, zeigt eine Flüssigkeit, welche beim Eintragen von $1 \mathrm{Aeq}$. Jod in concentrirte, 2 Aeq. KO enthaltende Kalilauge entsteht. Wasserstoffhyperoxyd entbindet aus letzterer stürmisch Sauerstoffgas und vernichtet sofort ihre Bleichkraft. Bei Zersetzung durch letzteres entsteht weniger jodsaures Kali und mehr Jodkalium, als bei freiwilliger Zersetzung. Diese Thatsachen führen zu dem Schlusse, dass sich das Jod zum Kali nicht anders verhalte als das Chlor; es entsteht erst unterjodigsaures Kali und Jodkalium, welches erstere alsdann, aber weit leichter und rascher, als es bei dem unterchlorigsauren Salz der Fall, in Jodkalium und jodsaures Kali zerfällt.

4. Eine Erklärung der auffallenden Thatsachen, dass die in 3 besprochene stark alkalische Flüssigkeit schon reinen Kleister, stärker noch jodkaliumhaltigen bläut, und Jodkaliumlösung bräunt, gibt der Verfasser noch nicht; jedenfalls aber stehen diese Reactionen im Zusammenhange mit den merkwürdigen Erscheinungen in $\mathbf{5}$.

5. $10 \mathrm{Grm}$. mit Jod gesättigten Wassers werden von 1 Tropfen eincr 10 Proc. Kali enthaltenden Kalilauge vollständig entfärbt, die Flässigkeit wird dabei alkalisch.- Versetzt man aber die 10 Grm. Jodlösung 
zuvor mit $10 \mathrm{Grm}$. 1 Proc. Stärke enthaltendem Stärkekleister, so wird das tief blaue Gemisch erst durch 4 Tropfen obiger Kalilösung entfärbt; setzt man dieser Mischung $0,1 \mathrm{Grm}$. Joolkalium zu, so bedarf sie zur Entfärbung 40, setzt man $0,5 \mathrm{Grm}$. Jodkalium zu 80, bei $1 \mathrm{Grm}$. 130 und bei 2 Grm. 160 Tropfen der besagten Kalilösung. - Aus diesen Thatsachen folgt, dass bei Anwesenheit von Jodkalium freies Jod und Kali neben einander bestehen können. Hiermit im Zusammenhange steht, dass die durch 4 Tropfen Kalilauge entfärbte Mischung von $10 \mathrm{Grm}$. Jodwasser und $10 \mathrm{Grm}$. Stärkekleister bei Zusatz von Jodkalium sich sofort wieder bläut, - sowie dass eine kalihaltige Jodkaliumlösung durch unterchlorigsaures Salz gebräunt, oder, wenn Stärkekleister zugegen, gebläut wird. - Es verliert jetzt auch die Ausscheidung des Jods aus Jodkalium oder die Bläuung des Jodkaliumkleisters durch Ozon ihr Auffallendes, denn man erkennt, dass bei Anwesenheit von Jodkaliumüberschuss das ausgeschiedene Jod neben dem entstandenen Kali existiren kann.

6. Versetzt man Jodwasser mit so viel Kalilauge, dass es für sich allein den ihm zugemischten Jodkaliumkleister nicht mehr zu bläuen "vermag, so thut es diess doch, sobald man in das Gemisch Kohlensäure einführt. Das jodsaure Kali nimmt hierbei nicht Theil, denn nach längerem Stehen oder nach dem Erhitzen der Flüssigkeit übt Kohlensäure diese Wirkung nicht mehr aus.

7. Wässrige Jodlösung, mit einer genügenden Menge Wasserstoffhyperoxyd vermischt, entfärbt sich sofort vollständig unter Bildung von Jodwasserstoffsäure. Man erkennt hieraus, dass Jodwasserstoff und Wasserstoffhyperoxyd, welche sich bei grösserer Concentration so leicht unter Jod-Ausscheidung zersetzen, bei starker Verdünnung neben einander existiren können.

8. Bringt man fein zertheiltes Jod mit Baryumhyperoxyd zusammen, welches frei von Baryt und in Wasser suspendirt ist, so verschwindet dasselbe rasch unter Entbindung gewöhnlichen Sauerstoffgases. Die Flüssigkeit enthält von jodsaurem Baryt freies Jodbaryum.

\section{Chemische Analyse anorganischer Körper. IVon}

R. Fresenius.

Bestimmung des Eisenoxyduls mittelst Chamäleons. Die diesen Gegenstand betreffende Abhandlung der Herren J. Löwenthal und 